\title{
Anatomical Study and Pollen Micromorphology of Onopordum L. in Iran
}

\author{
Elham AGHABABAEYAN ${ }^{1}$, Maneezheh PAKRAVAN ${ }^{1^{*}}$,
}

\author{
Farrokh GHAHREMANINEJAD ${ }^{2}$
}

${ }^{1}$ Alzahra University, Faculty of science, Department of Biology, Sheikh-Bahaee sq., Tehran, Iran, ${ }^{*}$ mpf176@yahoo.com ( ${ }^{*}$ corresponding author)
${ }^{2}$ Kharazmi University, Department of Plant Biology, Faculty of Biological Sciences, 43 Dr. Mofatteh Avenue, Tehran, Iran

\begin{abstract}
In this study, pollen micromorphological structure, leaf and stem anatomy structure of six species of Onopordum (O. acanthium L., O. armenum Grossh., O. carduchorum Bornm. \& Beauv., O. carmanicum (Bornm.) Bornm., O. heteracanthum C. A. Mey and $O$. leptolepis DC) were examined with different repetitions. About 17 qualitative anatomical features were statistically analyzed. Glandular trichome, cortex fiber tissue, embowed in subsidiary vascular, its fiber tissue and vascular sheath extensions, adaxial collenchyma tissue and types of adaxial and abaxial stomata were distinct characters in separating of the species. In palynologic studies based on P/E ratio, 2 types of pollen grains were observed: oblate- spheroidal and suboblate. SEM study of the exine showed echinate, reticulate and perforate sculpturing in all six species.
\end{abstract}

Keywords: Asteraceae, Iran, palynology, SEM, taxonomy

\section{Introduction}

The genus Onopordum L. belongs to Cardueae tribe of Asteraceae family, native to Europe (mainly the Mediterranean region), northern Africa, the Canary Islands, the Caucasus, and southwest and central Asia (Keleonikos, 2006). It has about 40 species in world which seven species has recorded from Iran (Rechinger, 1991; Aghababaeyan 2012), one including species native (Mozaffarian, 1996). This genus is resistant to hot weather and can grow in desert regions of Iran (Karimi, 2007). Fægri et al. (1989) have studied the pollen grain of Onoporum and combined pollen of Onopordum with those of the genera Saussurea, Arctium, Carlina and Carthamus in their Saussurea type on the features of blunt spines which the higth are as long as broad. Moore et al. (1991) included the pollen tyoe of Onopordum in the Serratula type. Valdés et al. (1987) placed the pollen of Onopordum in Senecio vulgaris type, a type containing many genera like Arctium, Carlina, Stahaelina. But Beug (2004) considered Cirsium, Doronicum, Senecio, $O$. acanthium and many others in the large Saussurea-Typ. The $O$. acanthium type is characterized by its broad, domeshaped echinae with small, subobtuse top and high digitate columellae of sexine which are distinctly higher in the equatorial plane than at the poles (Punt \& Hoen, 2009). Osman (2009) considered Onopordum and Atractylis in Onopordum pollen type.

The present study considers anatomy and micromorphological study of pollen in six Onopordum species occurring in Iran with the aim of providing some basic anatomical and palynological data and also to obtain the relationships between taxonomy in the one hand and anatomy and palynolog in the other hand.

\section{Materials and methods}

The plant materials were collected from the natural habitats in Iran (Tab. 1).

This study have performed on 40 populations of six Onopordum species, namely $O$. acanthium L., $O$. armenum Grossh., O. carduchorum Bornm. \& Beauv., O. carmanicum (Bornm.) Bornm., O. heteracanthum C. A. Mey. and $O$. leptolepis DC. (Tab.1). Anatomy of the leaf and stems, leaf epidermais and pollen morphology were examined. Leaves were fixed in $70 \%$ ethanol and stems in alcohol/glycerin (1:1). Cross sections of $1 / 3$ of the middle part of blade and third inter nod of the stem were used, double stained by methyl green and Carmine. Appropriate samples were photographed by light photomicroscope model $\mathrm{B} \times 51$. The epidermis was prepared using mixture of $\mathrm{H}_{2} \mathrm{O}_{2}$ and $5 \%$ sodium hydroxyl. The following equation was used to calculate the stomatal index (Cutter and Arnold, 1973):

$\mathrm{SI}=\mathrm{S} / \mathrm{E}+\mathrm{S}$ (SI :Stomatal index; S: guard cells; E: epidermal cells)

For studding the morphology of pollen, flowers were kept in a mixture of acetic acid 1:1 for 24 hours. The pollen were stained with Carmin and observed with ligth microscope. For scanning electron microscopy pollen were coated with a thin layer of gold. About 20-30 pollens were used in measuring polar axis, equatorial axis, colpus length, 
Tab. 1: Voucher specimen of Onopordum specimens. Abbreviations: ALUH: Alzahra University Herbarium.

\begin{tabular}{|c|c|c|c|}
\hline Species & Herbarium number & Address & Collector \\
\hline \multirow{12}{*}{ O. leptolepis } & ALUH, 11000 & Tehran, Gisha & Aghababaeyan \\
\hline & ALUH, 11001 & Tehran, Hesarak & Ghahremaninejad \\
\hline & ALUH, 11002 & Alborz, Golshahr & Aghababaeyan \\
\hline & ALUH, 11003 & Isfahan, Semirom & Aghababaeyan \\
\hline & ALUH, 11004 & Isfahan, 30km Shahreza & Aghababaeyan \\
\hline & ALUH, 11005 & Isfahan, $10 \mathrm{~km}$ Shahreza & Aghababaeyan \\
\hline & ALUH, 11006 & Isfahan, Shahreza & Aghababaeyan \\
\hline & ALUH, 11007 & Isfahan, Chadegan & Aghababaeyan \\
\hline & ALUH, 11008 & Isfahan, Najafabad & Aghababaeyan \\
\hline & ALUH, 11009 & Kohkiluye vabuyerahmad, Sisakht & Aghababaeyan \\
\hline & ALUH, 11010 & Fars, Dasht arjan & Aghakuchaki \\
\hline & ALUH, 11011 & Fars, Maharlu & Aghakuchaki \\
\hline \multirow{8}{*}{ O.armenum } & ALUH, 11012 & Karaj-Ghazvin road, 10km Ghazvin & Aghababaeyan \\
\hline & ALUH, 11013 & Gilvan-Zanjan road, $22 \mathrm{~km}$ Zanjan, Zaker & Aghababaeyan \\
\hline & ALUH, 11014 & Zanjan-Miane road, 6km Miane & Aghababaeyan \\
\hline & ALUH, 11015 & Zanjan-Ghazvin, $10 \mathrm{~km}$ Ghazvin & Aghababaeyan \\
\hline & ALUH, 11016 & Zanjan & Aghababaeyan \\
\hline & ALUH, 11017 & Azarbayejan sharghi, Ahar & Aghakuchaki \\
\hline & ALUH, 11018 & Azarbayejan sharghi, 8km Kalibar & Aghakuchaki \\
\hline & ALUH, 11020 & Isfahan, Najafabad, Azad university & Aghababaeyan \\
\hline \multirow{6}{*}{ O.heteracanthum } & ALUH, 11021 & Isfahan, Vilashahr & Aghababaeyan \\
\hline & ALUH, 11022 & Isfahan, Aliabad & Aghababaeyan \\
\hline & ALUH, 11023 & Isfahan, $40 \mathrm{~km}$ Chadegan & Aghababaeyan \\
\hline & ALUH, 11024 & Karaj-Ghazvin road, $70 \mathrm{~km}$ Ghazvin & Aghababaeyan \\
\hline & ALUH, 11025 & Karaj-Ghazvin road, 10km Ghazvin & Aghababaeyan \\
\hline & ALUH, 11026 & Kermanshah, Gilane gharb, Latechegha & Najafian \\
\hline \multirow[b]{6}{*}{ O. acanthium subsp. Araneosotomentosum } & ALUH, 11027 & Azarbayejan saharghi, Arasbaran & Pakravanfard \\
\hline & ALUH, 11028 & Markazi, Saveh & Ghahremaninejad \\
\hline & ALUH, 11029 & Isfahan, $60 \mathrm{~km}$ Naiin & Aghababaeyan \\
\hline & ALUH, 11030 & Isfahan, $10 \mathrm{~km}$ Naiin & Aghababaeyan \\
\hline & ALUH, 11031 & Yazd, Taft & Aghababaeyan \\
\hline & ALUH, 11033 & Zanjan & Aghababaeyan \\
\hline \multirow{4}{*}{ O. acanthium } & ALUH, 11034 & Azarbayejan sharghi, 8km Kalibar & Aghakuchaki \\
\hline & ALUH, 11035 & Ghazvin, Takestan & Tayebnejad \\
\hline & ALUH, 11032 & Gilvan-Zanjan, 50km Zanjan & Aghababaeyan \\
\hline & ALUH, 11036 & Azarbayejan sharghi, Bostanabad, Shebli & Tayebnejad \\
\hline \multirow{4}{*}{ O. carduchorum } & ALUH, 11037 & Gilan, Gilvan & Aghababaeyan \\
\hline & ALUH, 11038 & Lorestan, $25 \mathrm{~km}$ Poldokhtar & Aghababaeyan \\
\hline & ALUH, 11039 & Lorestan, $15 \mathrm{~km}$ Khoramabad & Aghababaeyan \\
\hline & ALUH, 11040 & Lorestan, Khoramabad & Aghababaeyan \\
\hline
\end{tabular}

pore diameter, exin thickness, number and length of spines. Spine numbers of the pollen were determined using the following equation (Christensen, 1986):

$\mathrm{N}=(\mathrm{P} /$ spines distance $) 2 \times \pi$

\section{Results and discussion}

Leaf and Stem: In transverse section of the leaf the midrib and upper surface was flat and the lower surface was convex (Fig. 2, 3).

A thick cuticle layer was observed on the outer surface of the stem epidermis. Several laminar layers of collenchymas were recognized under the epidermis. Under the collenchymas, parenchyma layers and in the central part, vascular bundles could be seen which were surrounded by sclerenchyma layers. Some trichomes were observed on epidermis which some of them were simple and others were glandular, which glandular trichomes in all taxa were sessile but in $O$. carmanicum were stalked (Fig. 4). The shape of crosse section of the leaves in all species except $O$. armenum were $U$ shape while in this taxon lower surface in subsidiary vascular bundles was flat (Fig. 3- I). O. acanthium $O O$. carduchorum and O. carmanicum have dorsiventral mesophyll and $O$. leptolepis, $O$. heteracanthum and $O$. armenum have isobilateral mesophyll (Fig. 3, 4). 


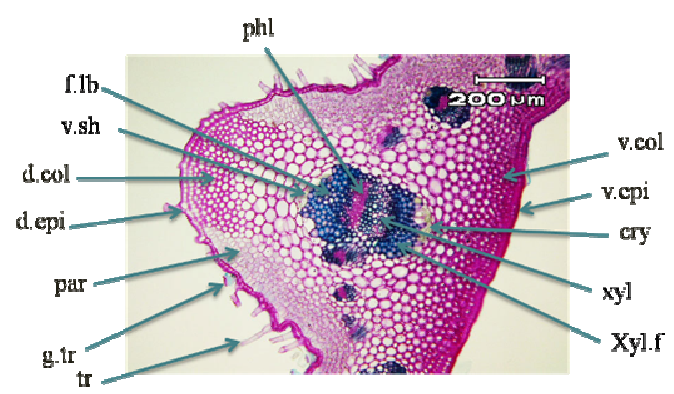

Fig. 2. Midrib of O. leptolepis in T.S. Tr: Trichome, G. Tr: Glandular Trichome, D. Epi: Dorsal Epiderm, D. Col: Dorsal Collenchyma, Par: Parenchyma, V. Sh: Vascular Sheath, F. Lb; Phloem Fiber, Phl: Phloem, Xyl: Xylem, Xyl. F: Xylem Fiber, Cry: Crystal, V. Col: Ventral Collenchyma, V. Epi: Ventral Epiderm. $(\mathrm{X}=60)$

Tab. 2. Anatomical qualitative characters of leaf in studied species.

\begin{tabular}{|c|c|c|c|c|c|c|c|}
\hline Species & O. acanthium & O. acanthium & O. carduchorum & O.armenum & O.leptolepis & O.heteracanthum & O.carmanicum \\
\hline $\begin{array}{c}\text { Transverse } \\
\text { section shape }\end{array}$ & U shape & U shape & U shape & - & U shape & U shape & U shape \\
\hline $\begin{array}{c}\text { convex in } \\
\text { subsidiary } \\
\text { vascular } \\
\text { bundle }\end{array}$ & * & - & * & - & * & * & * \\
\hline Mesophyll & Dorsiventral & dorsiventral & Dorsiventral & isobilateral & isobilateral & Isobilateral & dorsiventral \\
\hline $\begin{array}{l}\text { Glandular } \\
\text { trichome }\end{array}$ & sessile & sessile & Sessile & sessile & sessile & Sessile & stalked \\
\hline $\begin{array}{c}\text { Chrystal in } \\
\text { vascular } \\
\text { bundle }\end{array}$ & * & * & * & * & * & * & - \\
\hline $\begin{array}{l}\text { Chrystal in } \\
\text { mesophyll }\end{array}$ & * & * & - & * & * & - & - \\
\hline $\begin{array}{l}\text { Subsidiary } \\
\text { vascular } \\
\text { bundle fiber }\end{array}$ & $\begin{array}{c}\text { Xylem \& } \\
\text { phloem }\end{array}$ & phloem & $\begin{array}{c}\text { Xylem \& } \\
\text { phloem }\end{array}$ & - & $\begin{array}{c}\text { Xylem \& } \\
\text { phloem }\end{array}$ & Xylem \& phloem & Xylem \& phloem \\
\hline $\begin{array}{l}\text { Subsidiary } \\
\text { vascular } \\
\text { sheath } \\
\text { extension }\end{array}$ & $\begin{array}{l}\text { Upper \& } \\
\text { lower }\end{array}$ & upper & Upper \& lower & upper & $\begin{array}{c}\text { Upper \& } \\
\text { lower }\end{array}$ & Upper \& lower & Upper \& lower \\
\hline Cortex fiber & - & disport & Disport & continual & disport & Disport & disport \\
\hline
\end{tabular}

In $O$. carmanicum vascular fiber tissue completely surrounded the vascular bundle (Fig. 4-P) but in $O$. carduchorum these fibers were located in the upper and lower bundles (Fig. 3-D) and in $O$. armenum, $O$. leptolepis, $O$. heteracanthum and $O$. acanthium were in the upper and lower bundles or completely surrounded the vascular bundles (Fig. 3, 4). Xylem parenchyma was observed in all taxa. Vascular bundle sheath layer and it's extensions into the upper and lower side were similar in all taxa. Crystal was observed in vascular bundle sheath of all species except $O$. carmanicum (Fig. 4-P). Crystal in mesophyll cells was observed in $\mathrm{O}$. acanthium, $\mathrm{O}$. armenum and $O$. leptolepis (Fig. 3). Cortex fiber tissue continued in abaxial side in $O$. armenum (Fig. 3- I), but in $O$. acanthium was not observed (Fig. 3-B) and were disport in other species. In $O$. armenum subsidiary vascular bundles were without fiber (Fig. 3-C), this specie and $O$. acanthium subsp. araneosotomentosum had vascular sheath extensions in the upper side (Fig. 3-I). The quantitative traits: thickness of cuticle, epidermis, collenchymas, xylem and phloem fiber in central vascular bundle, xylem and phloem tissues, palisade and spongy parenchyma were measured (Tab. 5).

In a ventral and dorsal view the epidermal cells had flat walls. The anemocytic stomata occurred more frequently in the lower and upper epidermis. In addition the actinocytic stomata were observed on the lower epidermis in $O$. heteracanthum (Fig. 6-F) and upper epidermis in $O$. armenum and $O$. carmanicum

Fig. 5-B, D). The anisocytic stomata were observed on the upper epidermis in $O$. carduchorum and $O$. beteracanthum (Fig. 5-D, F). 

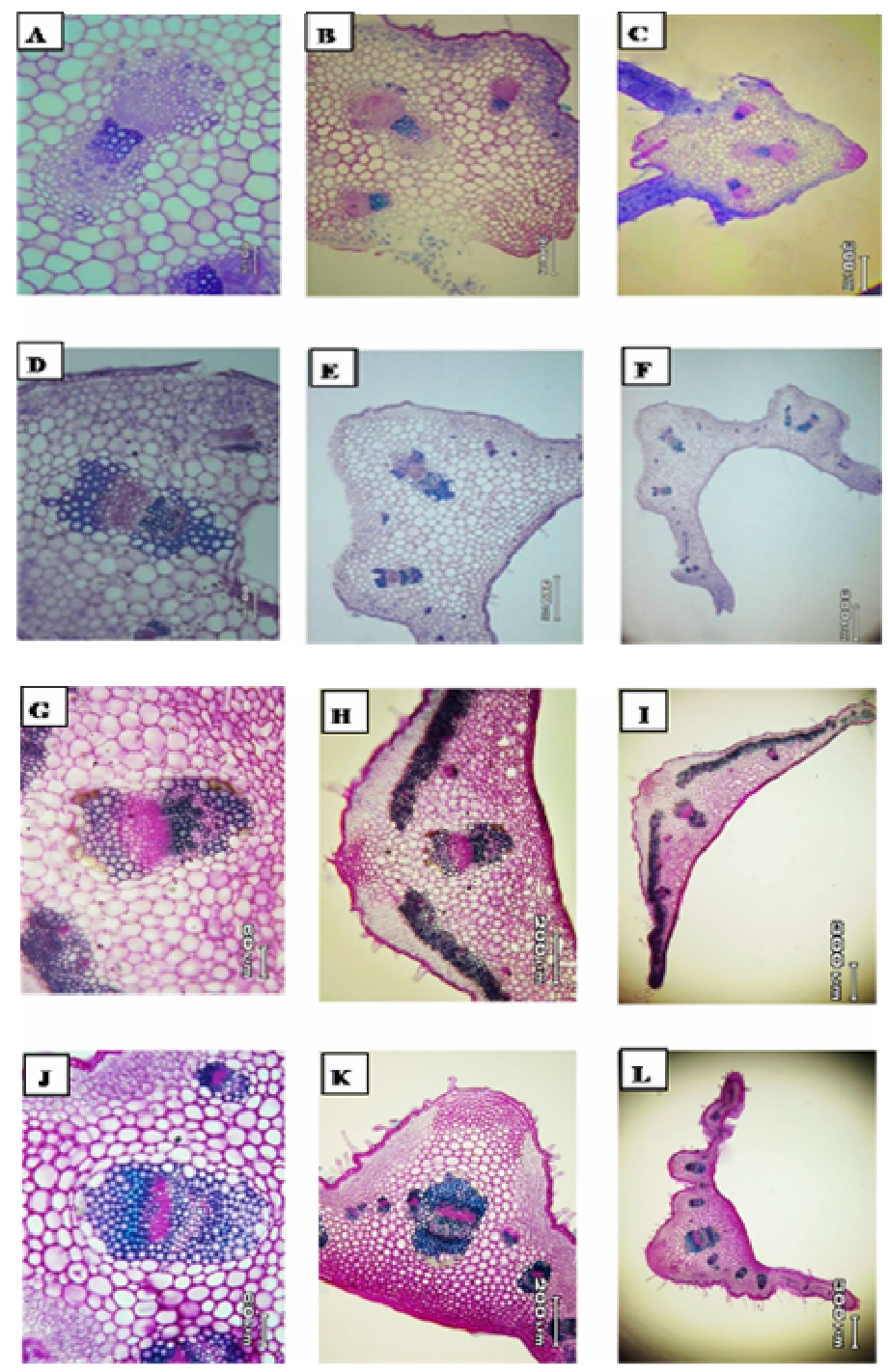

Fig. 3. Cross section of leaf in Onopordum species: (A-C): O. acanthium, (D-F): O. carduchorum, (G-I): O. armenum. (Scale bar $=200 \mu \mathrm{m}$ in B, E, K, H, X= 40; Scale bar=60 $\mu \mathrm{m}$ in A, G, D, J, X= 100; Scale bar=300 $\mu \mathrm{m}$ in C, F, I, L, X=20)
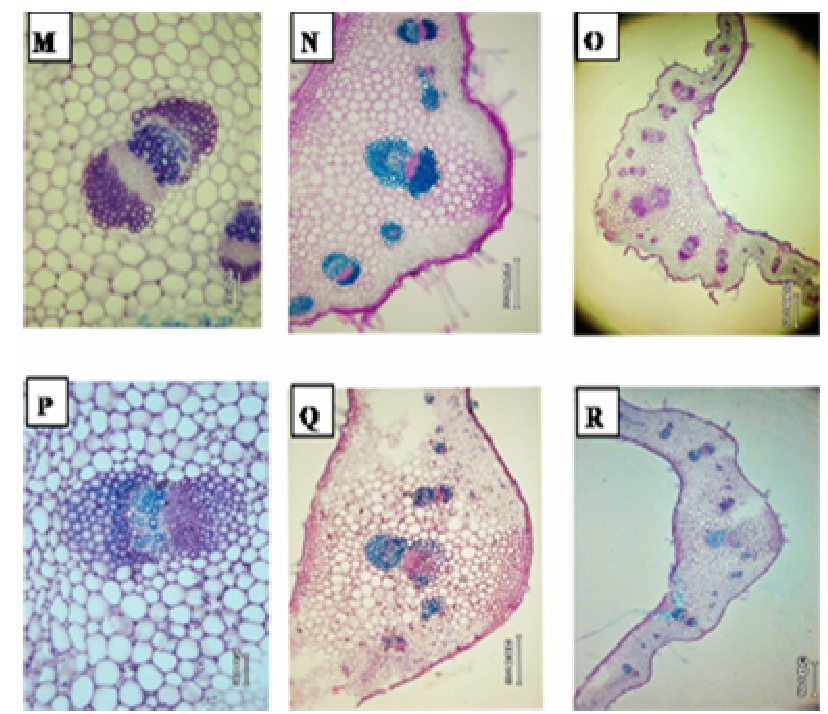

Fig. 4. Cross section of leaf in Onopordum species: (M-O): O. heteracanthum,(P-R): O. carmanicum. (Scale bar=60 $\mu \mathrm{m} \mu \mathrm{m}$ in $\mathrm{M}$, $\mathrm{P}, \mathrm{X}=100$; Scale bar=200 $\mu \mathrm{m}$ in $\mathrm{N}, \mathrm{Q}, \mathrm{X}=40$; Scale bar=300 $\mu \mathrm{m}$ in $\mathrm{O}, \mathrm{R}, \mathrm{X}=20$ ) 
70

Tab. 3. Anatomical quantitative characters of leaf in studied species (The unit is $\mu \mathrm{m}$ ).Le/Wid: Length of stem section/Width of stem section, Ad. Cu: Adaxial Cuticle's Thickness, Ab. Cu: Abaxial Cuticle's Thickness, Ad. Epi: Adaxial Epidermis Length, Ab. Epi: Abaxial Epidermis Length, Ad. Col. N: Adaxial Collenchyma Layer Number, Ab. Col. N: Abaxial Collenchyma Layer Number, Ad. Col. T: Adaxial Collenchymas Thickness, Ab. Col. T: Abaxial Collenchymas Thickness, Pal. Ad. N: Adaxial Palisade Layer Number, Pal. Ad. Le: Adaxial Palisade Cell Length, Pal. Ad. T: Adaxial Palisade Cell Thickness, Pal. Ab. N: Abaxial Palisade Layer Number, Spa. N: Spongy Layer Number, Spa. T: Spongy Thickness, Phl. F. N: Phloem Fiber Layer Number, Xyl. F. N: Xylem Fiber Layer Number, Phl. F. T: Phloem Fiber Thickness, Phl. T: Phloem Thickness, Xyl. T: Xylem Thickness.

\begin{tabular}{|c|c|c|c|c|c|c|c|}
\hline Characters & O. acanthium & $\begin{array}{l}\text { O. acanthium } \\
\text { subsp. }\end{array}$ & $\begin{array}{c}\text { O.. } \\
\text { carduchorum }\end{array}$ & $\begin{array}{c}O . \\
\text { armenum }\end{array}$ & $\begin{array}{c}O . \\
\text { leptolepis }\end{array}$ & $\begin{array}{c}O . \\
\text { heteracanthum }\end{array}$ & $\begin{array}{c}O . \\
\text { carmanicum }\end{array}$ \\
\hline Le/wid & 4.1 & 2.7 & 4.2 & 3.7 & 3.6 & 6.3 & 4.5 \\
\hline Ad.cu & 6.37 & 14.02 & 5.1 & 7.1 & 8.9 & 7.9 & 10.3 \\
\hline Ab.cu & 5.92 & 10.57 & 4.6 & 7.3 & 7.5 & 7.3 & 7.4 \\
\hline Ad.epi & 14.99 & 16.62 & 11 & 13.5 & 11.2 & 12.8 & 16.2 \\
\hline Ab.epi & 10.74 & 7.96 & 12.8 & 10.9 & 13.8 & 13.5 & 16.9 \\
\hline Ad.col.n & 1 & 1 & 1 & 1 & 1 & 2 & 1 \\
\hline Ab.col.n & 6 & 6 & 5 & 7 & 7 & 6 & 5 \\
\hline Ad.col.T & 19.66 & 17.82 & 17.3 & 16.6 & 21.7 & 28.6 & 23.9 \\
\hline Ab.col.T & 99.44 & 96.72 & 88.34 & 112.32 & 156.2 & 118.25 & 108.69 \\
\hline Pal,Ad.n & $2-3$ & $2 \_3$ & 2 & $2-3$ & $2-3$ & $2-3$ & 3 \\
\hline Pal.Ad.le & 33 & - & 41.8 & 31.35 & 27.8 & 27.3 & 29.1 \\
\hline Pal.Ad.T & 89.7 & - & 79.7 & 77.55 & 65.3 & 79.3 & 94.3 \\
\hline Pal.Ab.n & - & - & - & $1 \_3$ & $1 \_3$ & $2 \_3$ & - \\
\hline Spa.n & $4-7$ & 3 & $4-5$ & $2-4$ & $2-5$ & $4-5$ & $3-5$ \\
\hline Spa.T & 72.91 & 59.98 & 88.9 & 63.8 & 75.6 & 50.5 & 73.2 \\
\hline Phl.F.n & $6-9$ & 6 & 5 & $4-7$ & $4-10$ & $6-12$ & $5-12$ \\
\hline Xyl.F.n & 6 & 4 & 6 & $3-5$ & $3-10$ & $5-13$ & $5-9$ \\
\hline Phl.F.T & 93.04 & 73.8 & 78.9 & 69.5 & 91 & 129.8 & 134.2 \\
\hline Xyl.F.T & 83.94 & 41.3 & 81.5 & 58 & 92.4 & 113.3 & 111.4 \\
\hline Phl.F/Xyl.F & 1.17 & 1.7 & 0.9 & 1.3 & 1 & 1.1 & 1.1 \\
\hline Phl.T & 73.8 & 35.95 & 94.9 & 51.4 & 50.3 & 54.2 & 58.5 \\
\hline Xyl.T & 100.61 & 51.98 & 88.8 & 58.2 & 66.3 & 78 & 53.5 \\
\hline $\mathrm{Phl} / \mathrm{Xyl}$ & 0.73 & 0.69 & 1 & 0.9 & 0.7 & 0.5 & 0.7 \\
\hline
\end{tabular}

Cross section of the stem included cuticle layer, but glandular trichomes with stalk were just observed in trichomes, epidermal cells, parenchyma, collenchymas, O. carmanicum and O. carduchorum (Fig. 5-B, D). phloem and xylem fiber and vascular bundles (Fig.7). Crystals observed in vascular sheath of all the species The shape of cross section of the stem was angular in $O$. acanthium while were circular in other taxa (Fig. 5-A). Simple and glandular trichomes were observed in all taxa, except for $O$. carmanicum. Measured characters are shown in the Tab. 5.

Tab. 4. Stomatal character of leaf In Studied Species. LL: Lower Layer, LU: Upper Layer, SI: Stomata Index.

\begin{tabular}{cccc}
\hline Species & epidermis & Stomatal type & SI \\
O. acanthium & LL & Anemocytic & 6.7 \\
& LU & Anemocytic & 9.13 \\
O. carduchorum & LL & Anemocytic & 4.12 \\
O. armenum & LU & Anemocytic, Anizocytic & 4 \\
O. leptolepis & LU & Anemocytic & 7.1 \\
O. heteracanthum & LL & Anemocytic, Actinocytic & 8.4 \\
O. carmanicum & LU & Anemocytic & 13 \\
& LL & Anemocytic & 2.5 \\
& LU & Anemocytic, Actinocytic & 4.5 \\
\hline
\end{tabular}



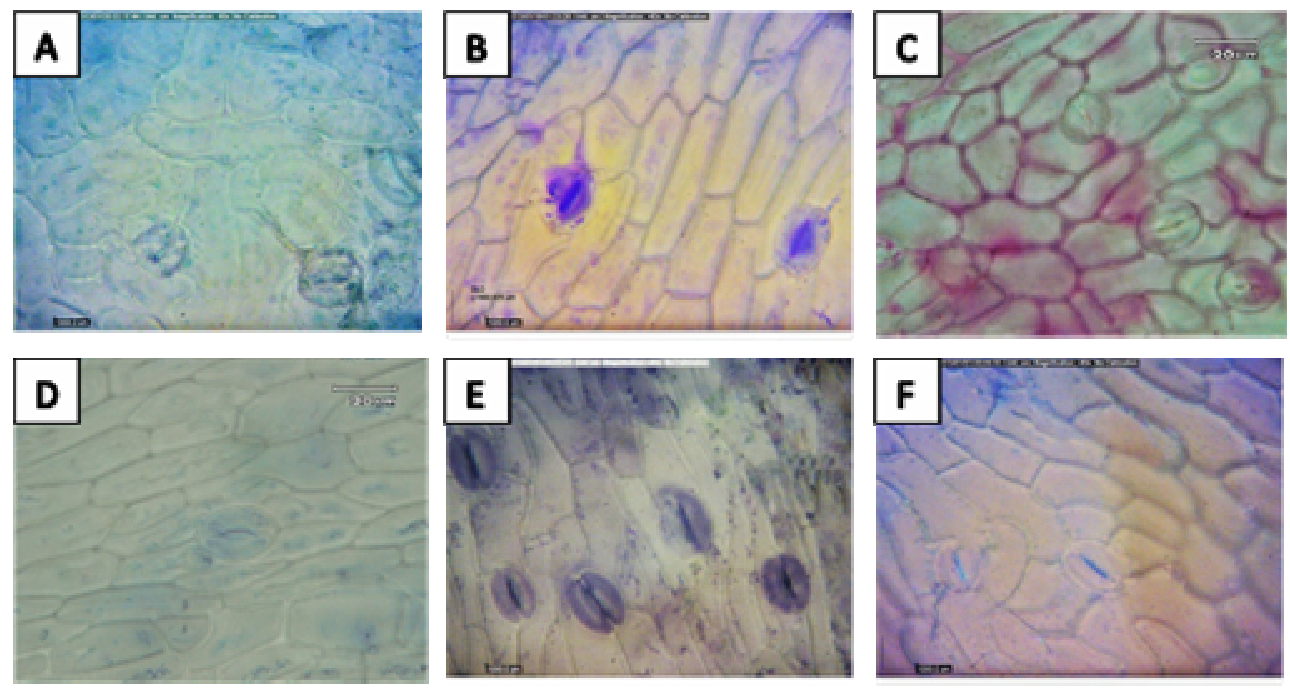

Fig. 5. Upper epidermis in : (A): O. acanthium; (B) : O. armenum; (C) :O. carmanicum; (D) :O. carduchorum; (E) : O. leptolepis; (F): O. heteracanthum. (Scale bar $=20 \mu \mathrm{m} ; \mathrm{X}=400)$
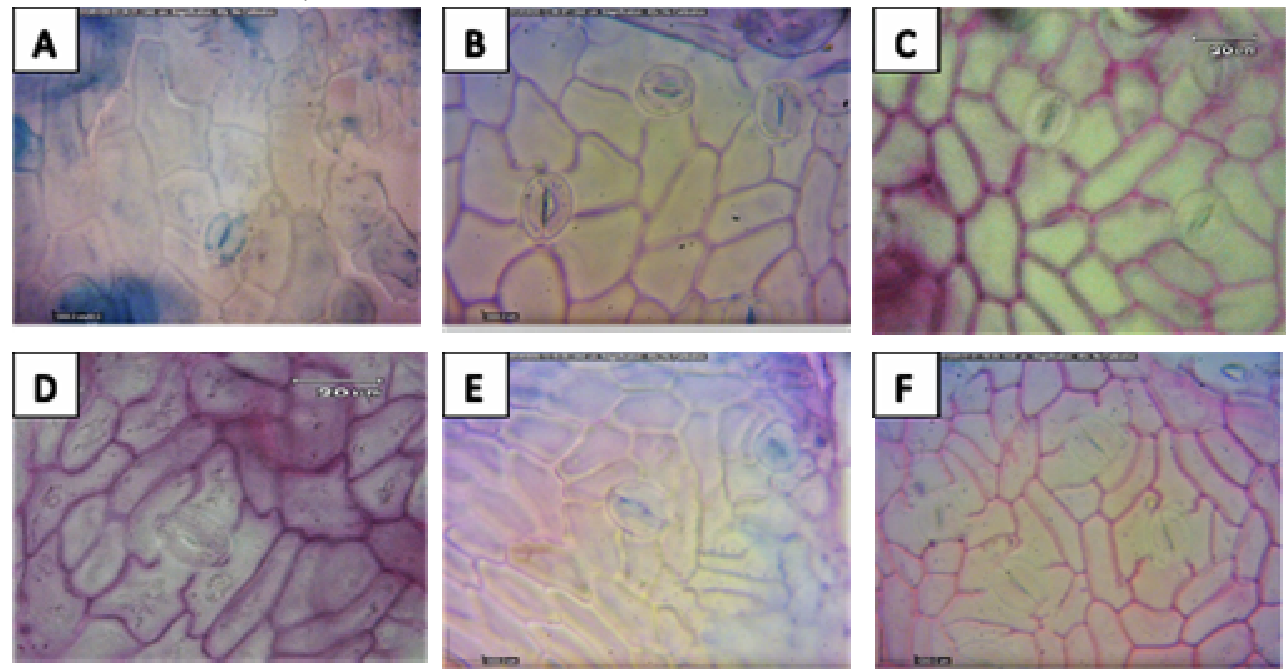

Fig. 6. Lowerl epidermis in: (A): O. acanthium; (B) :O.armenum; (C) :O. carmanicum; (D) :O. carduchorum; (E) :O. leptolepis; ( F) :O. heteracanthum. (Scale bar $=20 \mu \mathrm{m} ; \mathrm{X}=400)$

Tab. 5. Anatomical quantitative characters of stem in studied species (The unit is micrometer). Cu. T: Cuticle Thickness, Epi. T: Epidermis Thickness, Epi. Le: Epidermis Cell Length, Col. T: Collenchymas Thickness, P. C. T: Parenchyma Thickness, Ecl. Sh. T: Sclerenchymatous Sheaths Thickness, F. Lb. T: Phloem Fiber Thickness, Xyl. F. T: Xylem Fiber Thickness, Phl. T: Phloem Thickness, Xyl. T: Xylem Thickness.

\begin{tabular}{|c|c|c|c|c|c|c|c|}
\hline Species & O. acanthium & $\begin{array}{l}\text { O. acanthium } \\
\text { subsp. }\end{array}$ & O.carduchorum & O.armenum & O.leptolepis & O.heteracanthum & $\begin{array}{c}\text { O.carmanicu } \\
m\end{array}$ \\
\hline Cu.T & $\begin{array}{c}3.03-4.33 \\
3.87\end{array}$ & 4.02 & $\begin{array}{c}1.96-2.57 \\
2.35\end{array}$ & $\begin{array}{c}3.12-6.59 \\
4.52\end{array}$ & $\begin{array}{c}3.06-7.11 \\
4.84\end{array}$ & $\begin{array}{c}3.69-8.88 \\
5.57\end{array}$ & $\begin{array}{c}6.26-7.45 \\
7.06\end{array}$ \\
\hline Epi.T & $\begin{array}{c}13.55-15.69 \\
14.9\end{array}$ & 15.33 & $\begin{array}{c}10.81-15.7 \\
13.61\end{array}$ & $\begin{array}{c}9.6-17.25 \\
12.76\end{array}$ & $\begin{array}{c}10.41-18.12 \\
14.37\end{array}$ & $\begin{array}{c}11.16-23.39 \\
17.09\end{array}$ & $\begin{array}{c}15.43-18.04 \\
17.22\end{array}$ \\
\hline Epi.le & $\begin{array}{c}9.03-12.87 \\
10.64\end{array}$ & 6.83 & $\begin{array}{c}11.03-15.12 \\
12.67\end{array}$ & $\begin{array}{c}5.18-11.06 \\
8.5\end{array}$ & $\begin{array}{c}6.8-13.73 \\
9.33\end{array}$ & $\begin{array}{c}8.08-12.99 \\
10.66\end{array}$ & $\begin{array}{c}14.69-20.98 \\
17.2\end{array}$ \\
\hline Col.T & $\begin{array}{c}122.93-253.72 \\
188.92\end{array}$ & 73.77 & $\begin{array}{c}108.45-180.97 \\
146.67\end{array}$ & $\begin{array}{c}72.16-252.75 \\
164.4\end{array}$ & $\begin{array}{c}107.97-369.31 \\
201.44\end{array}$ & $\begin{array}{c}89.54-332.18 \\
204.98\end{array}$ & $\begin{array}{l}222.68- \\
373.09 \\
268.37\end{array}$ \\
\hline p.c. T & $\begin{array}{c}139.46-308.48 \\
188.52\end{array}$ & 130 & $\begin{array}{c}343.22-451.92 \\
575.79\end{array}$ & $\begin{array}{c}53.8-289.48 \\
169.17\end{array}$ & $\begin{array}{c}107.02-263.26 \\
204.89\end{array}$ & $\begin{array}{c}116.61-400.99 \\
233.52\end{array}$ & $\begin{array}{r}217.26- \\
494.36 \\
358.66\end{array}$ \\
\hline
\end{tabular}




\begin{tabular}{|c|c|c|c|c|c|c|c|}
\hline Ecl.sh.T & $\begin{array}{c}446.72-692.12 \\
557.75\end{array}$ & 570.1 & $\begin{array}{c}483.31-699.54 \\
575.79\end{array}$ & $\begin{array}{c}413.12-830.97 \\
593.73\end{array}$ & $\begin{array}{c}218.31- \\
1010.67 \\
764.22\end{array}$ & $\begin{array}{c}432.77- \\
1059.59 \\
661.73\end{array}$ & $\begin{array}{c}472.64-698.29 \\
607.82\end{array}$ \\
\hline f.lb.T & $\begin{array}{c}135.19-236.21 \\
191.13\end{array}$ & 182 & $\begin{array}{c}165.62-298.7 \\
229.95\end{array}$ & $\begin{array}{c}53.91-298.83 \\
177.88\end{array}$ & $\begin{array}{c}157.91-283.68 \\
221.07\end{array}$ & $\begin{array}{c}120.49-317.65 \\
206.05\end{array}$ & $\begin{array}{c}102.71-234.3 \\
170.09\end{array}$ \\
\hline Xyl.f.T & $\begin{array}{c}249-339.88 \\
233.84\end{array}$ & 172.8 & $\begin{array}{c}120.56-254.85 \\
220\end{array}$ & $\begin{array}{c}128.23-229.87 \\
173.41\end{array}$ & $\begin{array}{c}87.89-282.64 \\
217.77\end{array}$ & $\begin{array}{c}127.59-373.28 \\
228.1\end{array}$ & $\begin{array}{c}67.74-145.53 \\
110.01\end{array}$ \\
\hline f.lb.T/xyl.f.T & $\begin{array}{c}0.5-1.2 \\
0.77\end{array}$ & 1 & $\begin{array}{c}0.7-1.3 \\
1.02\end{array}$ & $\begin{array}{c}0.4-1.3 \\
0.96\end{array}$ & $\begin{array}{c}0.77-1.7 \\
1.01\end{array}$ & $\begin{array}{c}0.6-1.3 \\
0.92\end{array}$ & $\begin{array}{c}1.4-1.7 \\
1.5\end{array}$ \\
\hline Phl.T & $\begin{array}{c}57.78-96.72 \\
76.71\end{array}$ & 57.78 & $\begin{array}{c}64.26-111.37 \\
84.41\end{array}$ & $\begin{array}{c}43.17-129.94 \\
74.91\end{array}$ & $\begin{array}{c}44.89-103.07 \\
66.28\end{array}$ & $\begin{array}{c}40.52-82.19 \\
62.05\end{array}$ & $\begin{array}{c}60.66-94.87 \\
82.39\end{array}$ \\
\hline Xyl.T & $\begin{array}{c}100.49-266.65 \\
226.46\end{array}$ & 100.4 & $\begin{array}{c}109.26-243.75 \\
179.5\end{array}$ & $\begin{array}{c}98.16-176.21 \\
133.38\end{array}$ & $\begin{array}{c}102.85-339.65 \\
187.95\end{array}$ & $\begin{array}{c}99.79-210.09 \\
202.26\end{array}$ & $\begin{array}{c}111.02-192.76 \\
145.74\end{array}$ \\
\hline Phl.T/xyl.T & $\begin{array}{c}0.2-0.57 \\
0.33\end{array}$ & 0.57 & $\begin{array}{c}0.32-0.58 \\
0.47\end{array}$ & $\begin{array}{c}0.31-0.9 \\
0.53\end{array}$ & $\begin{array}{c}0.2-0.55 \\
0.35\end{array}$ & $\begin{array}{c}0.27-0.65 \\
0.42\end{array}$ & $\begin{array}{c}0.5-0.6 \\
0.53\end{array}$ \\
\hline
\end{tabular}

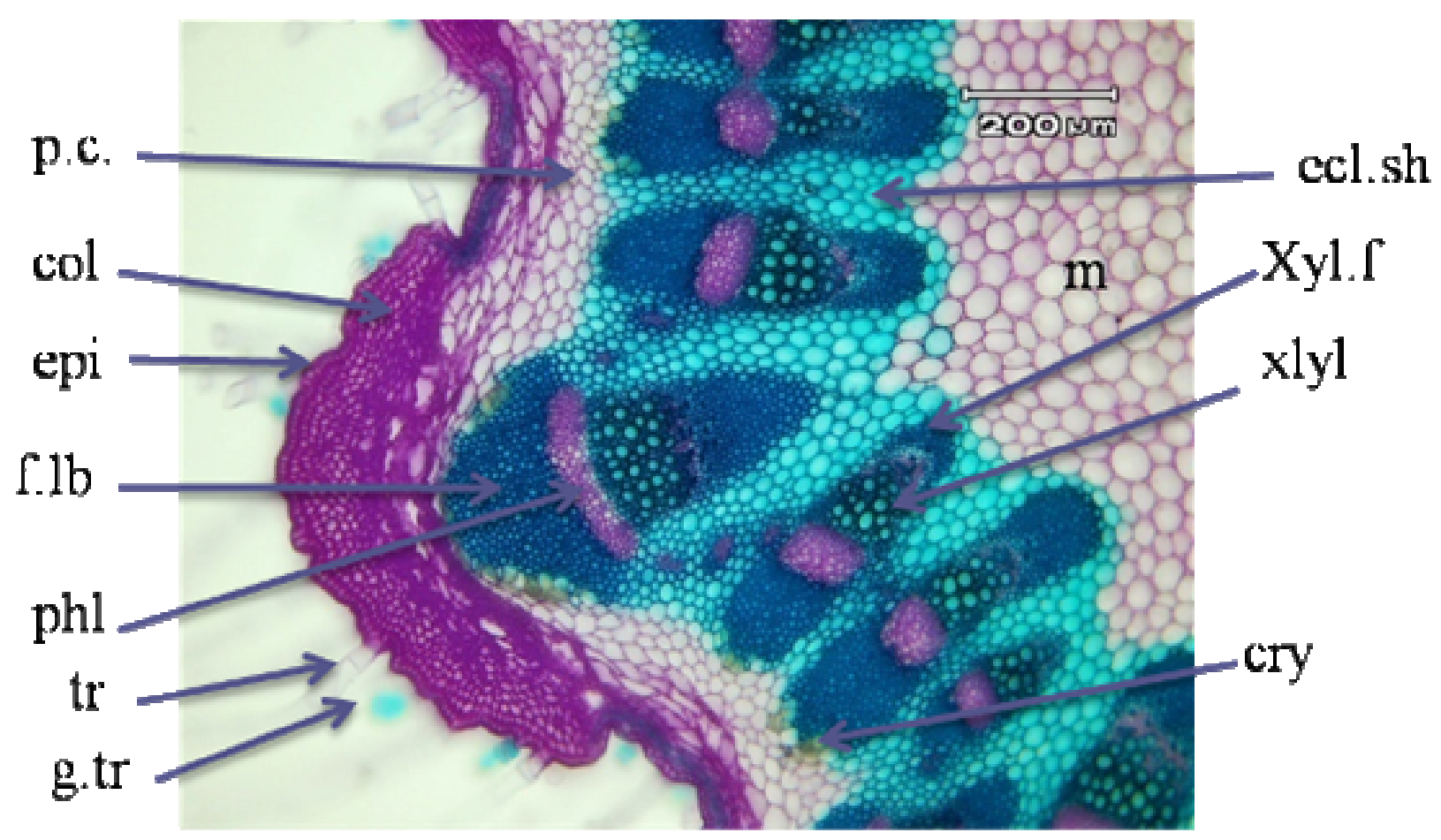

Fig. 7. The cross section of stem, in O. armenum. Tr: Trichome, G. Tr: Glandular Trichome, Epi: Epiderm, Col: Collenchyma, P. C: Parenchyma, Ecl. Sh: Sclerenchyma, F. Lb: Phloem Fiber, Phl: Phloem, Xyl; Xylem, Xul. F: Xylem Fiber, Cry: Crystal, M: Medulla. $00 \mu \mathrm{m}$ in B, E, K, H; Scale bar $=200 \mu \mathrm{m} ; \mathrm{X}=50$ ) 

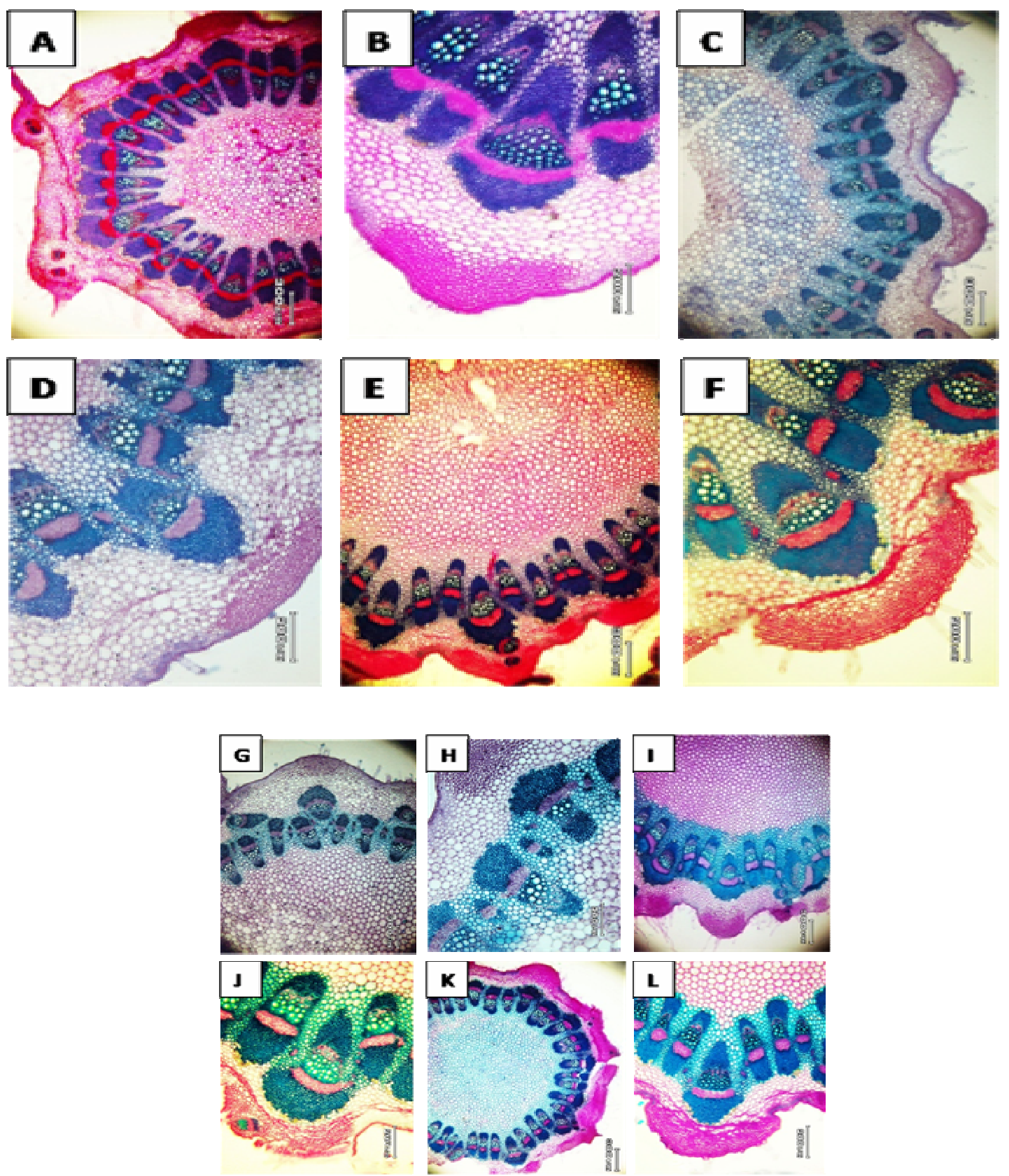

Fig. 8. Cross section of stem in Onopordum species: (A-B): O. acanthium; (C-D): O. carduchorum; (E-F): O. leptolepis; (G-H): O. carmanicum; (I-J): O. heteracanthum; (K-L): O. armenum. (Scale bar=200 $\mu \mathrm{m} ; \mathrm{B}, \mathrm{F}, \mathrm{H}, \mathrm{J}, \mathrm{L} ; \mathrm{X}=40$; (A, C, E, G, I, K: X= 20)

Pollen characters: All taxa had 3-zonocolporate pollen grains and Amb were rounded triangular or 3-lobate and elliptic equatorial view. According to $\mathrm{P} / \mathrm{E}$ ratio; $O$. acanthium and $O$. leptolepis were oblate-spheroidal and other suboblate (Tab. 6). Exin had echinate sculpture with the different length and number of spine in different species (Fig. 9). Heterobrochate sculpture in $O$. carduchorum and $O$. heteracanthum, microreticulate sculpture in $O$. acanthium and perforate sculpture in $O$. carmanicum and O. leptolepis were observed (Fig. 8). Lumen with $0.2-1.26 \mu \mathrm{m}$ diameter in $O$. carduchorum, with $0.44-1.1 .55 \mu \mathrm{m}$ diameter in $O$. heteracanthum and with in $0.3-0.53 \mu \mathrm{m} O$. acanthium were observed. Perforate diameter in O. leptolepis were $0.2-0.76 \mu \mathrm{m}$ and in O. carmanicum $0.26-0.46 \mu \mathrm{m}$ (Tab. 6). 

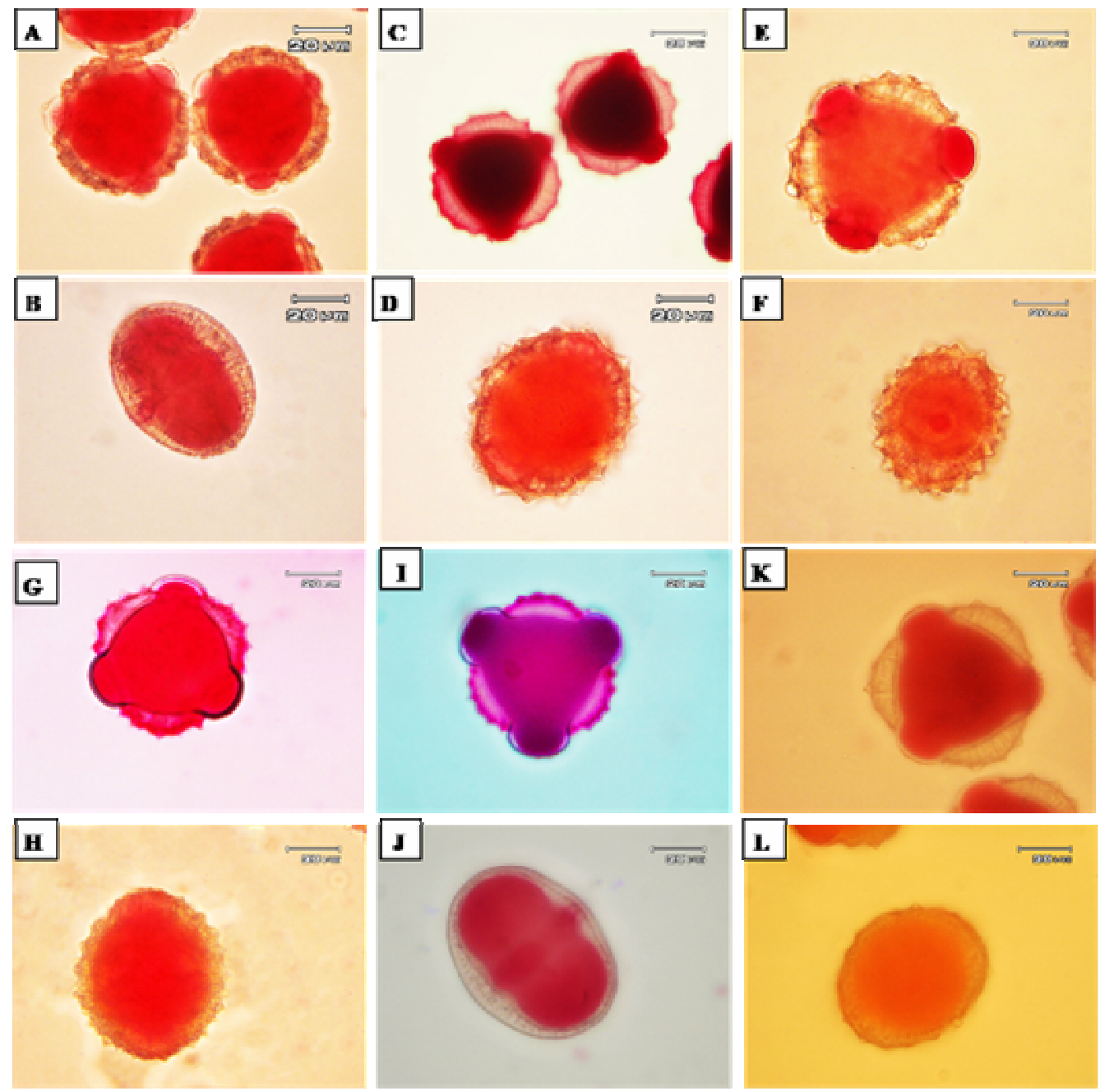

Fig. 8. Pollen grains in Onopordum species by light microscope. (A, B): O. acanthium, (C, D): O. carduchorum, (E, F): $O$. armenum, $(\mathrm{G}, \mathrm{H})$ : O. leptolepis, $(\mathrm{I}, \mathrm{J}):$ O. heteracanthum, $(\mathrm{K}, \mathrm{L}):$ O. carmanicum. (Scale bar $=20 \mu \mathrm{m}, \mathrm{X}=400$ )

Tab. 6. Tabular summary showing the pollen grains dimensions (The unit is micrometer). P: Polar axis, E: Equatorial axis, Cl: Colpus Length, Po. Di: Pore Diameter, Et: Exin Thickness, N. Sp: Number of Spines, Le. Sp: Length of Spines, OS: Oblate Spheroidal, SO: Suboblate.

\begin{tabular}{|c|c|c|c|c|c|c|c|}
\hline Characters & Species & O. acanthium & O. carduchorum & O. armenum & O. leptolepis & O. heteracanthum & O. carmanicum \\
\hline \multirow{3}{*}{$P$} & Min & 43.57 & 41.27 & 48.1 & 49.31 & 51.68 & 45.29 \\
\hline & Mean & 45.93 & 51.62 & 55.05 & 55.99 & 52.63 & 49.62 \\
\hline & Max & 47.84 & 63.53 & 51.19 & 59 & 53.59 & 59.52 \\
\hline \multirow{3}{*}{$\mathrm{E}$} & Min & 47.3 & 56.43 & 59.32 & 58.18 & 59.3 & 56.47 \\
\hline & Mean & 50.37 & 66.41 & 63.54 & 62.28 & 63.03 & 58.11 \\
\hline & Max & 53.85 & 71.12 & 66.66 & 66.55 & 66.71 & 60.74 \\
\hline \multicolumn{2}{|c|}{$\mathrm{P} / \mathrm{E}$} & 0.91 & 0.77 & 0.86 & 0.89 & 0.83 & 0.85 \\
\hline \multicolumn{2}{|c|}{ shape } & OS & So & So & OS & So & So \\
\hline \multicolumn{2}{|c|}{$\mathrm{CL}$} & 52.50 & 66.68 & 39.93 & 50 & 54.19 & 53.76 \\
\hline \multicolumn{2}{|c|}{ Po.di } & 15.88 & 23.36 & 19.02 & 22.28 & 20.33 & 20 \\
\hline \multicolumn{2}{|l|}{ ET } & 6.8 & 10.89 & 11.86 & 11.21 & 10.25 & 10.58 \\
\hline \multicolumn{2}{|c|}{ N.Sp } & 100 & 135 & 148 & 153 & 104 & 99 \\
\hline \multicolumn{2}{|c|}{ Le.Sp } & 1.7 & 1.44 & 4.18 & 3.99 & 3.13 & 2.5 \\
\hline
\end{tabular}


75

Phenetic analysis: In order to find most variable traits, principal component analysis was implemented. Primitive analysis showed that three first factors were responsible for the $80 \%$ of total studied variation in taxa. In the first factor with almost $35 \%$ of the total variation, leaf cross section shape, lower surface in subsidiary vascular bundles and vascular sheath extensions had the highest positive correlations. In the second factor with nearly $24 \%$ of observed variation, cortex fiber and subsidiary vascular bundles fiber had the highest positive correlation. In the third factor with $20 \%$ of total variation, upper leaf collenchymas and leaf stomata had the highest positive correlations.

By studding cluster analysis using anatomical characters (Ward method) showed the clear separation of the species studied (Fig.1). Two clusters were observed. The first cluster was composed of $O$. acanthium, $O$. leptolepis, $O$. heteracanthum and $O$. armenum. The species of $O$. carduchorum and O. carmanicum, were positioned in the second cluster. $O$. acanthium and $O$. leptolepis had similar stomata on their ventral epidermis and crystals in their mesophyll cells, they had also oblate-spheroidal pollens. $O$. acanthium had minimum number of pollen grain, pore diameter and exin thickness, this species was the only one with angular shape of transverse section of the stem. Results revealed that $O$. acanthium and $O$. leptolepis were closely related, they have similar stomata on ventral epidermis. Dendrogram showed the close relationships between $O$. carmanicum and $O$. carduchorum, both having glandular trichomes with stalk on their stems. $O$. armenum was located in a separate cluster by itself, because of cortex fiber tissue, cross section shape, lower surface in subsidiary vascular bundles and subsidiary vascular bundles fiber.

\section{Dendrogram using torar Method}

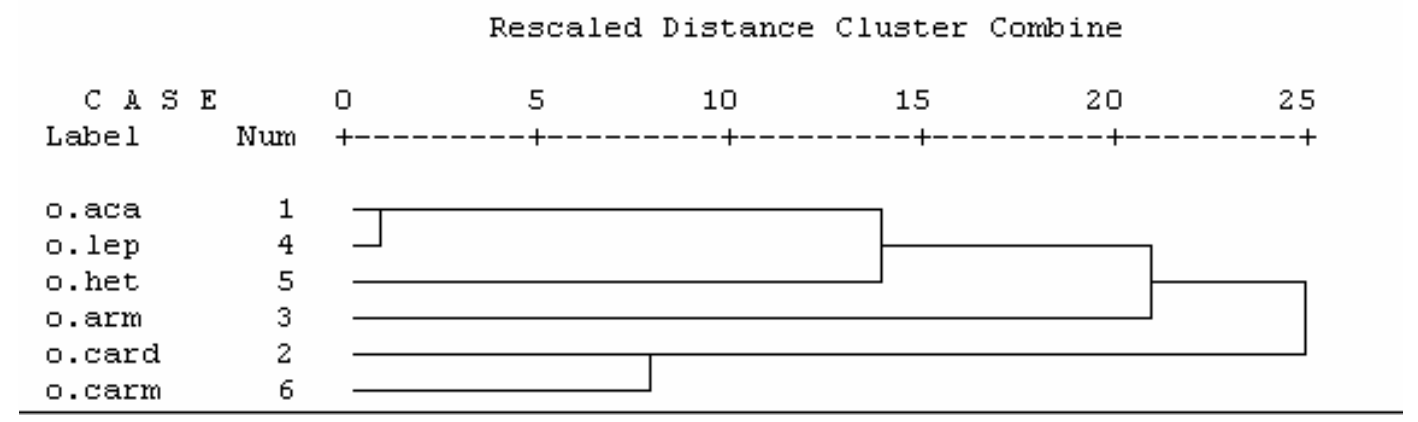

Fig.1: Dendrogram by Ward method for Onopordum species studied. 1: O. acanthium, 2: O. carduchorum, 3: O. armenum, 4: $O$. leptolepis, 5: O. heteracanthum, 6: O. carmanicum.
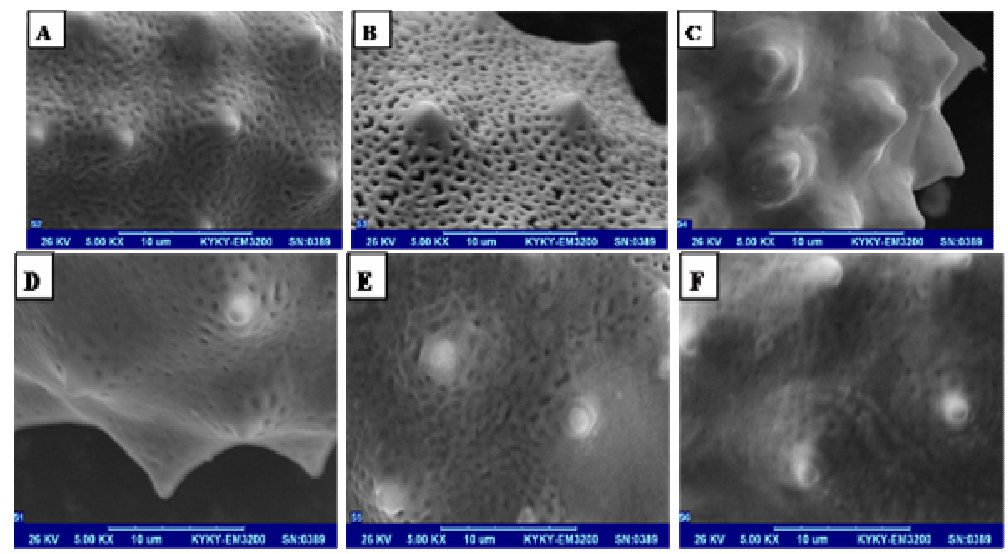

Fig. 9. SEM picture of exin in species of Onoporpum: (A): O. acanthium, (B): O. carduchorum, (C): O. armenum, (D): O. leptolepis, (E): O. heteracanthum, (F): O. carmanicum. $(\mathrm{X}=3600)$

Our results showed that in anatomical study, $O$. armenum could be easily identified because of certain characters that has mentioned above. $O$. armenum comprised a separate cluster by itself, more over this species was the only species without microreticulate or perforate sculpture of the pollens, also it had the maximum length of spines. $O$. carduchorum and $O$. carmanicum had glandular trichomes with stalk, similar number of abaxial collenchymas layers, and convex midrib in abaxial surface, phloem thickness and xylem thickness. Pollens were suboblate in both species and the exine thickness was similar. They could be distinguished from each other by maximum polar axis, maximum equatorial axis, colpus diameter and pore diameter (Table 6). 
We concluded that $O$. acanthium and $O$. leptolepis are closely related species and $O$. armenum is an independent species that is separated from other species.

Also it has shown that anatomical characters such as leaf shape in cross section, subsidary vascular bundles in leaf, vascular sheath, presence of fiber and subsidary vascular bundles fiber in cortex, stomata type and index, presence of crystal, mesophyll type, trichome type in leaf and stem were diagnostic characters in the genus Onopordum. More over P/E ratio, lumen diameter and number of spine were useful characters in distinguishing the species.

\section{References}

Aghababaeyan E (2012). Systematic study of the genus Onopordum L. (Asteraceae) from Iran. unpublished Msc. Thesise, Alzahra University, Tehran.

Beug HJ (2004). Leitfaden der Pollenbestimmung für Mitteleuropa und angrenznde Gebiete. Verlag Dr. Friederich Pfeil, München, p. 542.

Christensen PB (1986). Pollen morphological studies in the Malvaceae, Grana. 25:95-117.

Cutter EG, Arnold, E (1973). Plant anatomy: experiment and interpretation part I. Cell Tiss. Spring.
Fægri K, Kaland PE, Krzywinski K (1989). Textbook of Pollen Analysis, 4th Ed. Wiley, Chischester. p. 328.

Karimi H (2007). A dictionary of Iran's vegetations (plants). Vol.IV. Parcham, Tehran (in Persian).

Kleonikos GS, (2006). Wild edible plants of Crete. Rethymnon Crete, ISBN 960-631-179-1.

Moore PD, Webb JA, Collinson ME (1991). Pollen Analysis. Second Edition. Blackwell Scientific Publishers, Oxford. p. 216.

Mozaffarian V (1996). A dictionary of Iranian plant name. Farhang Mo'asser, Tehran (in Persian).

Osman AK (2009). Contributions to the pollen morphology of Tribe Cardueae (Cichorioideae-Compositae). Fedd Repert 120:145-157.

Punt W, Hoen PP (2009). The northwest European Pollen flora, 70 Asteraceae-Asteroideae. Rev Paleobot Palyno 157:22-183.

Rechinger KH, Dittrich M, Petrak F, Wangenitz G (1991). Compositae III._Cynareae in Rechinger K. H. (ed.), Flora Iranica, Compositeae III, 139-a. Acad. Druck.u.Verlastantal. Graz.

Valdés B, Díez MJ, Fernández I (1987). Atlas polinico de Andalucia Occidental. Universidad de Sevilla Excma. Diputacion de Cadiz. p. 450. 